Electrical Impedance Tomography With a Lab-on-Chip for Imaging Cells in Culture

Peer-reviewed author version

LEMMENS, Marijn; Biesmans, Hanne; BORMANS, Seppe; VANDENRYT, Thijs \& THOELEN, Ronald (2018) Electrical Impedance Tomography With a Lab-on-Chip for Imaging Cells in Culture. In: PHYSICA STATUS SOLIDI A-APPLICATIONS AND MATERIALS SCIENCE 2015 (15), (Art No 1700868).

DOI: $10.1002 /$ pssa.201700868

Handle: http://hdl.handle.net/1942/26652 


\title{
Electrical impedance tomography with a lab-on-chip for imaging cells in culture
}

\author{
Marijn Lemmens $^{1}$, Hanne Biesmans ${ }^{1}$, Seppe Bormans ${ }^{1}$, Thijs Vandenryt ${ }^{1}$, Ronald Thoelen ${ }^{1,2}$ \\ ${ }^{1}$ Hasselt University, Institute for Materials Research, Wetenschapspark 1, B-3590 Diepenbeek, Belgium \\ 2 IMEC vZw, IMOMEC Division, Wetenschapspark 1, B-3590 Diepenbeek, Belgium
}

Received ZZZ, revised ZZZ, accepted ZZZ

Published online ZZZ (Dates will be provided by the publisher.)

Keywords Tomography, Impedance, Cell imaging, Cell proliferation

\begin{abstract}
Electrical Impedance Tomography (EIT) is a non-invasive, non-ionizing and inexpensive imaging modality that is used to image the conductivity distribution inside the subject under test. EIT is an emerging imaging technique that has the potential to be used in a variety of (bio)medical applications. A technology that is easy to integrate into a small portable device and also easy to setup. In this work a custom made impedance analyser is used as a measurement device. The working principle is based on the different conductivity distributions of the material under test, this due to inhomogeneous bioelectrical properties. How-
\end{abstract}

\begin{abstract}
ever there is one major downside of this technique, the reconstruction problem of EIT is severely ill-posed. This means that the definition of a correct model is essential. Because of this ill-posed condition, a comparison of different models is done. In this work, an in depth study is performed to achieve the most optimal way of solving the inverse problem, which leads to noise suppression and reproducible results. This technology, integrated in a lab-onchip for monitoring cellular growth, is based on a spatial reconstructed imaging technique using electrical impedance tomography.
\end{abstract}

1 Introduction Recent studies have shown the applicability of electrical impedance tomography (EIT) in (bio)medical imaging. One of the most convenient applications of EIT is monitoring the thorax. It is a radiation-free imaging modality, that can be used at the bedside with patients. The technique is suggested for the assessment of haemodynamic events such as stroke volume, blood pressure and pulmonary perfusion [1]. The inhomogeneous bioelectrical properties will cause an inhomogeneous conductivity distribution. This feature can be used to form tomographical images.

In Regenerative Medicine (RM), imaging is imperative for the characterisation purposes as well as monitoring the progression of the regeneration. Many types of imaging techniques already exist in the translational research, these techniques often rely on synthetic or genetic fluorescent labels. However this labelling technique can be far from ideal, when looking at exogenous contrast labels, like nanoparticles, the reliability will decay over time. Using genetically encoded labels will compensate for this. These labels have a good short-term as well as long-term lifespan, yet in human setting there are regulatory issues relating to the safety of genomic integration [2]. For this, EIT can be used as a valid alternative. Because the physics of the probing energy on the excitation electrode is diffusive, EIT is much more sensitive to conductivity changes near the electrodes than elsewhere. So as a consequence of this finding the correct solution for the reconstruction problem is one of the major challenges due to the ill-conditioned property of this reconstruction problem. Because of this ill-posed condition, a comparison of different models is made to verify the influence of different approaches to solve the reconstruction formulation.

In this paper, a planar EIT sensor is developed to monitor cell proliferation. By using yeast cells, a proof-of-concept setup is created to monitor the cell proliferation. The sensor consists out of a circular array pattern of electrodes, placed inside a well. This gives the ability to inject a current into the medium and measure the changes in potential values on the boundary electrodes. The sensor that consists out of a custom made impedance analyser, has the ability for switching between multiple channels, accurate voltage measurement and has the capability to generate a current with a low noise floor [3]-[5]. 


\section{Reconstruction algorithms}

2.1 Principle of EIT The EIT reconstruction technique is in essence finding the solution for a nonlinear problem by reconstructing the conductivity map in a closed domain $(\Omega)$ based on the surface potential data developed at the boundary by injecting a constant current signal, see equation (3). The mathematical theory of EIT can be split up into two parts: the "forward problem" and the "inverse problem" [6]. The forward solver tries to determine the boundary potential data and this for a known current injection pattern. The inverse solver calculates conductivity distribution for which the boundary voltage difference becomes minimum, see equation (1).

$$
\Delta V=V_{m}-V_{c}=V_{m}-f
$$

Where $V_{m}$ stands for the measured potential value at the boundary and $V_{c}$ is the calculated one. The parameter $f$ represents a function that maps a vector that has a length equal to the amount of elements, from which the model exists, into a vector consisting out of the total number of measured voltages $V_{m}$.

The next step is to find a relation between the electrical conductivity value $(\sigma)$ of each point inside the area of interest and their corresponding potential values $(\Phi)$. This relation can be represented by a derived formula from the Maxwell's equation.

$$
\nabla \cdot(\sigma \nabla \Phi)=0
$$

As equation (2) represents a nonlinear partial differential equation it is not that trivial to solve it. In other words, since the potential distribution is dependent on the conductivity distribution, a direct or analytical method of solving will fail. This problem can be overcome by using a numerical technique like finite element method (FEM). By discretising the area of interest into small elements, an approximation of the correct values can be determined. However, this raises the following problem, the ill-posed problem of the EIT, equation (2) will have an infinite number of possible solutions if it is not provided with boundary conditions [7], [8]. In EIT the FEM technique is used to find the relationship between the developed potential differences in the area of interest and the current that is injected. This relationship is mathematically described as the Jacobian $(J)$, represented by equation (3). Based on the Geselowitz's sensitivity theorem (Geselowitz et al, 1971) a sensitivity method for fast calculation of the sensitivity map is provided. The theorem provided an implementation of impedance sensitivity of a fourpoint measurement.

$$
J=\oint_{\Omega} \nabla \Phi_{s} \cdot \nabla \Phi_{d} d(\Omega)
$$

In this equation (3) $\Phi_{s}$ and $\Phi_{d}$ stand respectively for the forward solution regarding the potential distribution for a specific source and the forwards solution for the adjoint source. In essence the forward model tries to find the relation between the injected current matrix $(C)$ and the potential value matrix $(\Phi)$ found at the electrodes. This relationship is established through the transformation matrix $(K(\sigma))$. So based on equation (4), the forward solver has to find a solution for the potential value matrix $(\Phi)$.

$$
[\Phi]=[K(\sigma)]^{-1}[C]
$$

The inverse solver, in contrary to the forward solver, tries to find the conductivity distribution matrix $(\sigma)$ when the current matrix $(C)$ and potential value matrix $(\Phi)$ are known. The goal is to find the minimal difference between the simulated potential values $(\Phi)$ and the boundary data vector $(\Delta V)$. This is done by finding the least square solution of the function that is represented in equation (5).

$$
s=\frac{1}{2}\left\|V_{m}-f\right\|^{2}=\frac{1}{2}\left(V_{m}-f\right)^{T}\left(V_{m}-f\right)
$$

However, equation (5) results into an ill-posed problem. To overcome this, a regularization method is included in the equation which redefines the object function $s$ into a wellposed problem. This can be described by the following general framework, expressed by equation (6).

$$
\min _{\sigma}\left\{\frac{1}{2}\left\|V_{m}-f\right\|^{2}+\lambda\|G(\sigma)\|^{2}\right\}
$$

Where the matrix $(G(\sigma))$ stands for the regularization term. Looking at a more practical approach of the inverse problem, equation (6) can be solved iteratively by using the GaussNewton method. So starting originally from equation (5), after differentiating, the general equation for EIT is found [6]. The algorithm starts by having an initial guess of the conductivity matrix $\left(\sigma_{k}\right)$ and is updated with a conductivity update vector $(\Delta \sigma)$ for every iteration. Using the following matrix $\left(\sigma_{k+1}\right)$, the forward solver finds a new potential distribution matrix $(\Phi)$ based on equation (4). Both voltage mismatch vectors $\left(\Delta V_{k}\right)$ and $\left(\Delta V_{k+1}\right)$ are compared to each other until a minimum is found. This results into equation (7), expressed in $(\mathrm{S} / \mathrm{m})$, that represents the solution for the conductivity distribution for every iteration $k$.

$$
\sigma_{k+1}=\sigma_{k}+\left(\left(J^{T} J+\lambda I\right)^{-1}\left(J^{T}\left(V_{m}-f\right)-\lambda I \sigma\right)\right)_{k}
$$

So the inverse process works in the opposite way when compared to the forward solver. This means that the inverse solver tries to find a solution for the conductivity distribution matrix $(\sigma)$ when the current matrix $(C)$ and the potential value matrix $(\Phi)$ are known. 


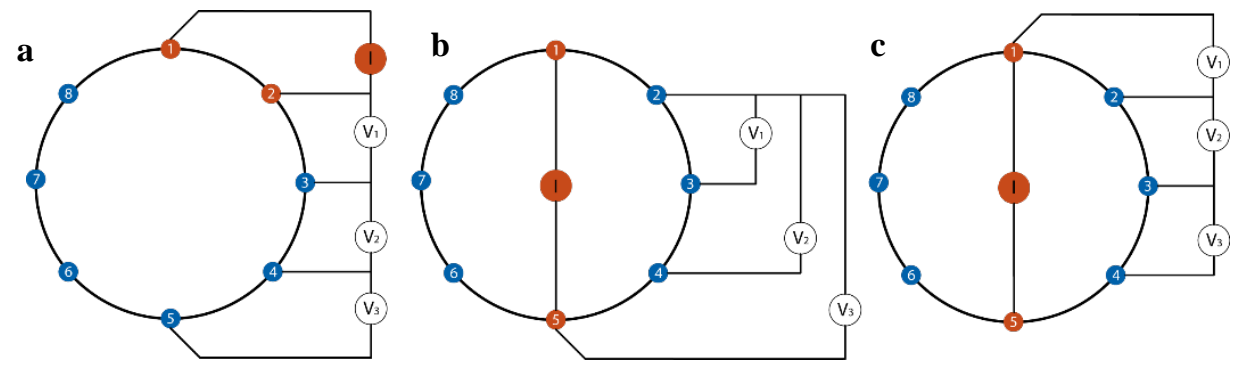

Figure 1 Schematic diagram showing the principle of EIT for the first three measurement of the first cycle. (a) the adjacent current projection method. (b) The opposite current projection method. (c) The opposite driving/adjacent measuring method.

To summarise the EIT algorithm works in the following 8 steps:

1. Calculate the boundary voltage values $\left(V_{c}\right)$ for a known current vector $(C)$ and an initial guess for the conductivity matrix $\left(\sigma_{k}\right)$ in the first iteration.

2. The voltage mismatch vector $\left(\Delta V_{k}\right)$ is calculated by comparing the measured voltage values $\left(V_{m}\right)$ and the calculated voltage values $\left(V_{c}\right)$.

3. After finding the potential distribution matrix $(\Phi)$ the Jacobian $(J)$ is calculated.

4. The update conductivity vector $(\Delta \sigma)$ can be calculated by using the Gauss-Newton iterative method.

5. The conductivity matrix $\left(\sigma_{k+1}\right)$ is regenerated by adding the update conductivity vector $(\Delta \sigma)$ to the previous conductivity vector $\left(\sigma_{k}\right)$.

6. The new conductivity matrix $\left(\sigma_{k+1}\right)$ is used in the forward solver to find a new voltage mismatch vector $\left(\Delta V_{k}\right)$.

7. Check if the voltage mismatch vector $\left(\Delta V_{k}\right)$ satisfies the requirements of the specified threshold for the error margin.

8. If the voltage mismatch vector $\left(\Delta V_{k}\right)$ meets the requirements, the solution for the conductivity value matrix $\left(\sigma_{k}\right)$ is found. Hence, the image can be created based on these conductivity values. If the criteria is not met, the algorithm starts back from the top until the pre-set threshold is minimally achieved.

2.2 Measurement patterns In EIT there are several ways of measuring techniques, the boundary potential values and injecting patterns of current can be done in various ways. In this research, the three most common methods are tested: adjacent, opposite and a combination of the previous methods.

Starting with the adjacent method (also known as the neighbouring method or the Sheffield data collection protocol) [9]. In this method, the current is injected in two neighbouring electrodes and the voltage is measured from other successive adjacent electrode pairs. More concrete, in the first cycle of measurements, the current is injected in E1-E2 and the voltage is measured from the electrode pairs E2-E3, E3-
E4, E4-E5, E5-E6, E6-E7, E7-E8, E8-E1. Note that electrode-pair E1-E2 was excluded from voltage measurements, but E2-E3 and E8-E1 were not, see figure (1a). The next cycle of measurements is one with current injection on E2E3 and voltage measurements from E3-E4, ..., E1-E2. After 8 cycles of measurements, there are 56 voltage measurements available [10].

The second one is the opposite method as proposed by Hua et al. With this method, the current is injected in opposed electrodes. So firstly, the current is injected in electrode pair E1-E5. For the voltage measurements, E2 is taken as the reference electrode, so voltage is measured from E2-E3, E2E4, E2-E5, E2-E6, E2-E7, E2-E8 and E2-E1, see figure (1b). Subsequently, the electrode-pair E2-E6 becomes the new current injecting pair and E3 the new reference electrode for the voltage measurements. Also here the process is repeated 8 times, so in the end there are 56 measurements collected [10].

The third method is a combination of the two above. It will be addressed as the opposite driving/adjacent measuring method in this paper. In this method, the current will be injected on opposite electrodes, but the voltage measurements will be performed on adjacent electrodes. This means that in the first cycle the current is injected in electrode pair E1-E5 and the voltage measurements will happen from E1-E2, E2E3, E3-E4, E4-E5, E5-E6, E6-E7, E7-E8 and E8-E, see figure (1c). In the next cycle, everything shift an electrode pair, so the current is injected in E2-E6 and the measurements take place on E2-E3, ..., E1-E2. In the end, there is a dataset with 64 data points available.

2.3 Hyperparameter selection One of the most important decisions to make when talking about the reconstruction algorithm is the way of determining the regularisation parameter, also called the hyperparameter $(\lambda)$. This parameter is able to choose a ratio between the image resolution and the smoothing of the data. Selecting this value could be done on a heuristic manner by reconstructing different images with each a different hyperparameter and select the best image [11]. However, this method is subjective 
and hard to reproduce [12]. A better approach for this problem is to calculate the hyperparameter based on different algorithms. In the next section the four ways to calculate this parameter will be explained.

The current state-of-the-art techniques to select the appropriate regularization hyperparameter consists out of four methods that are commonly used [13].

The first way to determine the hyperparameter $(\lambda)$ is called the fixed noise figure (NF). The noise figure was firstly introduced by Adler and Guardo and is defined as the ratio of the signal-to-noise-ratio (SNR) in the measurements to the SNR in the image, see equation (8).

$$
N F=\frac{S N R_{\text {measurements }}}{S N R_{\text {image }}}=\frac{\frac{\operatorname{mean}(m)}{\sqrt{\operatorname{var}(n)}}}{\frac{\operatorname{mean}(S)}{\sqrt{\operatorname{var}(S n)}}}
$$

With the input signal $m$, the input noise $n$ and the output signal $S$. A NF value is picked and the algorithm then automatically calculates the corresponding $\lambda[14]$.

Secondly, there is the most recent method to predict the hyperparameter $\lambda$, this method is called the image $\overline{S N R}$ [13]. The method determines the expected SNR in the image as a measure for the noise performance (NP). The SNR is determined for different targets and the average SNR over all the targets is the final NP metric. Once the final $\overline{S N R}$ value is calculated, a bisection search technique was applied to calculate the value of $\lambda$.

$$
\overline{S N R}=\frac{1}{n_{t}} \sum_{i=1}^{n_{t}} S N R_{i}
$$

Thirdly, the use of the L-curve approach is looked at. In this approach, the regularized solution is plotted parametrically against the corresponding residual vector. Both the values have to be as small as possible for an ideal hyperparameter value. Normally this plot has an L-shape. The value in the curvature of the $L$ is the most correct value for $\lambda$, because this is the place where both of the values are the smallest [15]. Hence this point represents the best compromise between the data mismatch and the regularization parameter $\lambda$. This means that the L-curve displays the trade-off between minimizing the residual norm $\left(\left\|V_{m}-f\right\|_{2}^{2}\right)$ and the regularization term $\left(\|G(\sigma)\|_{2}\right)$ for a certain $\lambda$, see equation (6) [16].

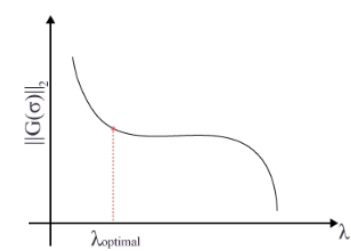

Figure 2 A typical plot of the L-curve to find the most optimal value of $\lambda$. The values are obtained by plotting all the solutions over the full range of $\lambda$ values.
Finally, a last method for determining the regularization parameter the Generalized Cross Validation method (GCV) is used. This method is based on the principle that if one element of the dataset is left out, the GCV-function $\operatorname{GCV}(\lambda)$ should be able to predict this value, however only if the right value of $\lambda$ is chosen [17].

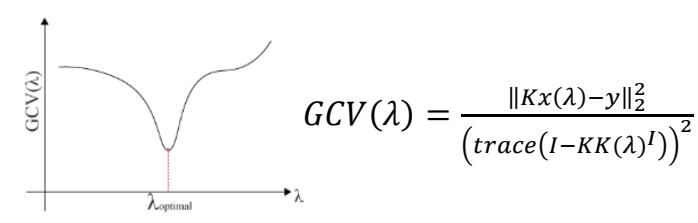

Figure 3 The lowest cross-validation error is the optimal solution for $\lambda$, this is represented by the lowest value of the GCV-function.

Where in equation (11) $K(\lambda)^{I}$ stands for a transformation matrix which will map the measured data $(y)$ onto the regularized solution $x(\lambda)$, i.e., $x(\lambda)=K(\lambda)^{I} y[15]$.

2.4 Prior selection Image priors are powerful tools to improve the quality of the reconstructed images, but it is important to keep in mind that they are based on probabilistic assumptions. Therefore, it is possible that some priors are not suitable for every case and it is very important to remain critical [18]. The three used priors will be briefly quoted below.

The first prior that was used was the Laplace prior. This is a second order high pass filter which takes the inter-elements correlations in consideration by calculating the penalty term in a finite element model for each element and for his adjacent elements. On a finite element mesh, a value of -1 is assigned to the neighboring element, a value of 0 to the elements that do not border on the element and the element itself gets a value of $(D+1)$, where $D$ stands for the model dimension and is equal to three because of the $3 \mathrm{D}$ model that is used in this paper. The approach of this common edge sensitive filter makes it possible to detect edges and to make the reconstruction of the phantom more demarcated [18].

The second and really popular prior is the Tikhonov regularization parameter. The use of the regularization term can be seen in equation (6), by adding this term to the least square minimization function it becomes a well-posed expression with a unique solution for $\lambda>0$. The Tikhonov parameter will exclude sudden variations and also smoothens the data. This results into a more stable problem [19].

The third prior was the Newton One Step Error Reconstructor (NOSER). This method takes one step of the Newton method, uses an estimated $\sigma_{0}$ and keeps it constant. These calculations can be done analytically. The NOSER algorithm also includes a modification of the Jacobian matrix. This is necessary because it is ill-conditioned what means that it has both large and small eigenvalues. The small eigenvalues would, if the Jacobian matrix is inverted, become large eigenvalues and those will cause large errors [20]. 


\section{Materials and methods}

3.1 Cross-validation of the models Before diving into the development of the application, a test setup is prepared to cross-validate all of the above mentioned solver, hyperparameter selection and prior selection. By doing this the reliability of the system can be evaluated.

3.2 Test setup To do the cross-validation of the models the following test device is made. It consists of a polymethyl methacrylate (PMMA) container with a height of 6.0 $\mathrm{mm}$ and diameter of $5.5 \mathrm{~cm}$. The sensor consists out of 8 evenly spaced electrodes in a circular pattern fixed on a printed circuit board (PCB). The PCB was covered with a $2.0 \mathrm{~mm}$ thick polydimethylsiloxane (PDMS) protective layer from which the electrodes extended.

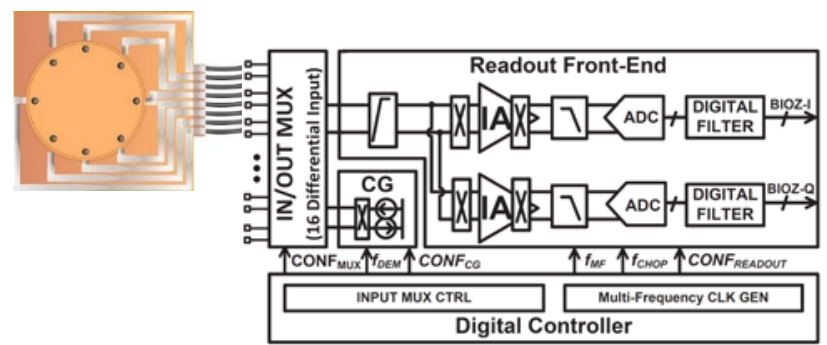

Figure 4 A simplified overview of the test setup were the device is connected to a schematic representation of the impedance analyser (MUSEIC V2.0) [2].

When placing an object with a different conductivity as the medium into the container, the resulting EIT image should show a difference in conductivity at the same place the object is placed. For these first tests an iron object is placed into the container, because the metal object is more conductive then the $0.9 \% \mathrm{NaCl}$ medium the resulting image should show a higher conductivity area around the place of the metal object.

3.3 Sensor setup Besides the test device a different setup is created to monitor the growth curve of yeast. The sensor contained 8 electrodes that were evenly spaced in a circular pattern with a radius of $1.00 \mathrm{~cm}$ on a PCB. In order, to culture the yeast, two layers of PDMS $(3.0 \mathrm{~mm})$ with a square hole $(2.00 \mathrm{~cm} \times 2.00 \mathrm{~cm})$ were placed on top of each other on the PCB. To prevent leaking, the PDMS was taped to the PCB with double-sided tape (Tesa AG 4900, Acryl transfertape). Finally, a sheet of polymethyl methacrylate
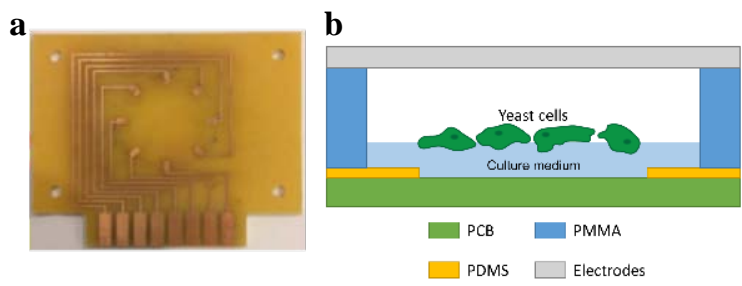

Figure 5 (a) The sensor that is used to measure the growth curve of yeast. (b) A cross-section of the sensor.
(PMMA) is placed on top of the well to eliminate any evaporation.

To monitor the growth of yeast, Saccharomyces cerevisiae $(0.02 \mathrm{~g} / \mathrm{ml})$ was used. As a culture medium a $10 \%$ glucose solution was used, this supports the cell proliferation. First the medium was added in the sensor and afterwards the yeast cells were placed on top. These yeast cells will dissolve into the medium and subsequently will precipitate. The measurements were conducted in a temperature controlled incubator, it was set at a constant temperature of $30.0{ }^{\circ} \mathrm{C}$. The yeast sensor was placed in the incubator for a time span of 24 hours, every 56 seconds a picture was taken with a camera together with EIT measurements. This was done so that after the measurements were conducted a visual reference image was available to compare the eventual generated EIT images with.

3.4 System instrumentation The measurements are performed with the MUSEIC V2.0, a low-power sensor with multiplexing capabilities [3]. In this research, the adjacent method is used with the driving electrodes included, using this method gives a total of 56 potential values for 8 electrodes. The duration of measuring all the potential values and the switching of the multiplexer takes in total $56.0 \mathrm{~s}$, for switching between the channels it takes $0.5 \mathrm{~s}$ and again another $0.5 \mathrm{~s}$ for the measurement itself. Every measurement was done with the following parameters: a current of $5 \mu \mathrm{A}$, a frequency of $8 \mathrm{kHz}$ and a gain of $70 \mathrm{~V} / \mathrm{V}$.

To test the setup, a conductive object is placed into the container, as a result the inverse problem should give an indication of the placement of the objects locations as a change in conductivity at the particular location. With the sensor containing an 8 electrode setup a total of 56 measurements are made. These measurements, combined with a known current that is send into the area of interest and therefor by extend into the unknown medium, are the input elements that the inverse problem needs. The output will be a $2 \mathrm{D}$ image that represents the conductivity values in a planar surface.

\section{Results and discussion}

4.1 Validation measurements In figure (6) an overview is given of the measurements that are conducted with the test setup, here the influence of different settings in the mathematical model, which are described earlier, are shown. Overall, the reconstructed images were correct if the comparison is made with the placement position of the object. There was an anomaly visible around electrode number four, but since this anomaly was present in almost all different combinations this was probably a result of bad contact resistance of the electrode number four. All the solutions resulted into a good image and there was no obvious difference between the methods to calculate the hyperparameter $(\lambda)$, this can be explained because of the large difference in conductivity values between the medium and the conductive 


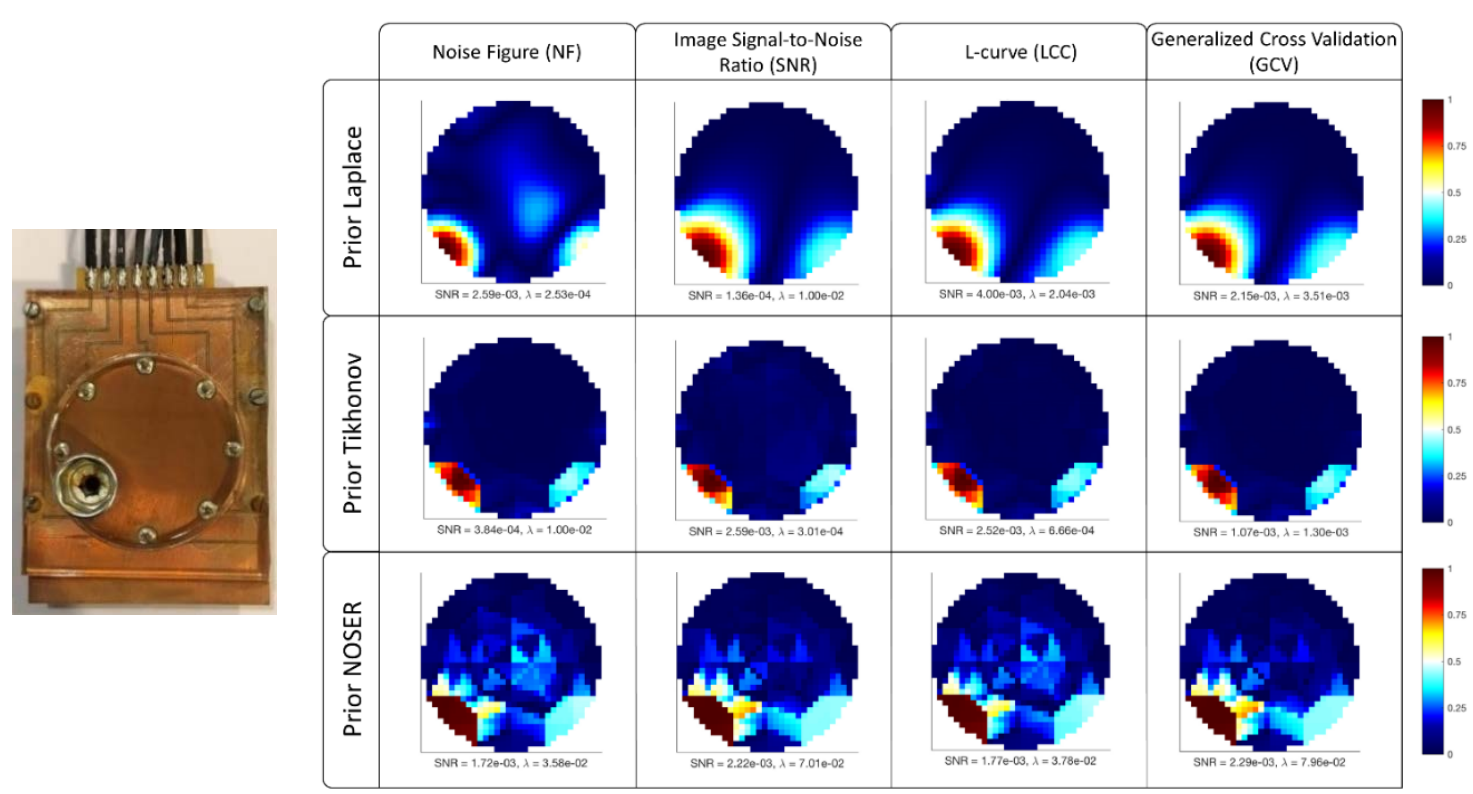

Figure 6 Resulting images when placing a conductive object near electrode 4 . The red area near this electrode indicates the presence of a conductive object near this electrode. The conductivity values are normalized between 0 and 1 , this is done because only an indication is needed that shows the correct position of a conductive object.

object. When other measurements are conducted, where the conductivity values are closer together, the different methods for calculating the hyperparameter $(\lambda)$ can help to determine a more suitable hyperparameter value. Something that stands out by looking at the images is the difference in resolution. This is due to the different priors. For the Laplace prior the detection of rapid intensity changes is clearly visible in the images. When the Tikhonov prior is used in combination with the Gauss-Newton method, the reconstruction process is stabilized and discontinuities in the image are smoothened. Finally, the NOSER prior is used, here a clear drop of resolution is visible. Because this prior alters the Jacobian matrix to keep a balance between the resolution and the data smoothness, the low resolution is a consequence of the modified Jacobian matrix to find a suitable solution.

4.2 Experimental measurements To know if it is even possible to detect the cellular growth of yeast the impedance spectra of agar and a combination of agar and yeast is measured.

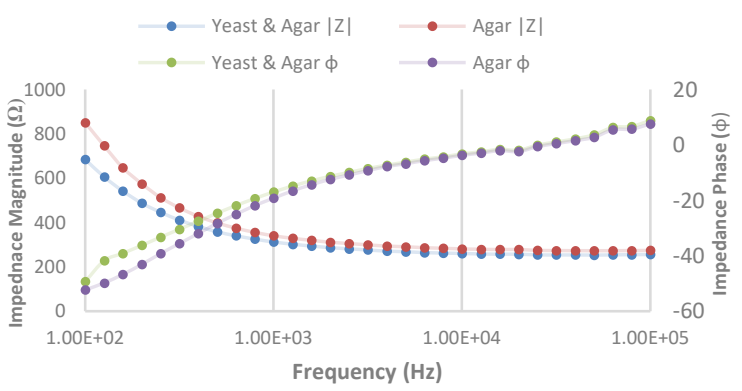

Figure 7 Both the magnitude and phase are shown for a $1 \%$ agar gel solution and a mixture of agar and yeast.

The current electrodes are put on the first and second electrode of the sensor while the sensing electrodes are put on the opposite electrodes (5 and 6), see figure (1).
A clear difference is noticeable between them in impedance values, so by extension the conductivity values. After looking at this result it is possible to state that it is possible to see the growth of the yeast cells into agar by using the EIT imaging technique.

4.3 Monitoring the culture of yeast Before the solver can calculate the corresponding images of the measured boundary potential values, an accurate model of the physical setup is required. To do this the framework of EIDORS is used in MATLAB (MathWorks, Inc., USA), a compilation of algorithms that falls under the GNU Public License [18]. The model represents a well with a diameter of $1.00 \mathrm{~cm}$ that is filled with $0.40 \mathrm{~cm}$ of medium. This 3D model however is not the model that is used in the inverse problem, instead of solving this model a simplified alternative of this model is taken. The medium, is assumed to be constant in the $z$ direction. Leaving out these variabilities result into a reconstruction of a $2 \mathrm{D}$ image. An additional difficulty when using the principle of EIT on cellular growth are the minimalistic changes of impedance during the process of growing the cells. This section describes a two-way approach on imaging to address this problem.

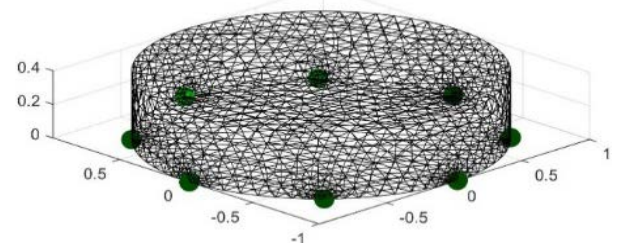

Figure 8 The 3D model used in the forward model: height $=0.40 \mathrm{~cm}$ and diameter $=1.00 \mathrm{~cm}$. 


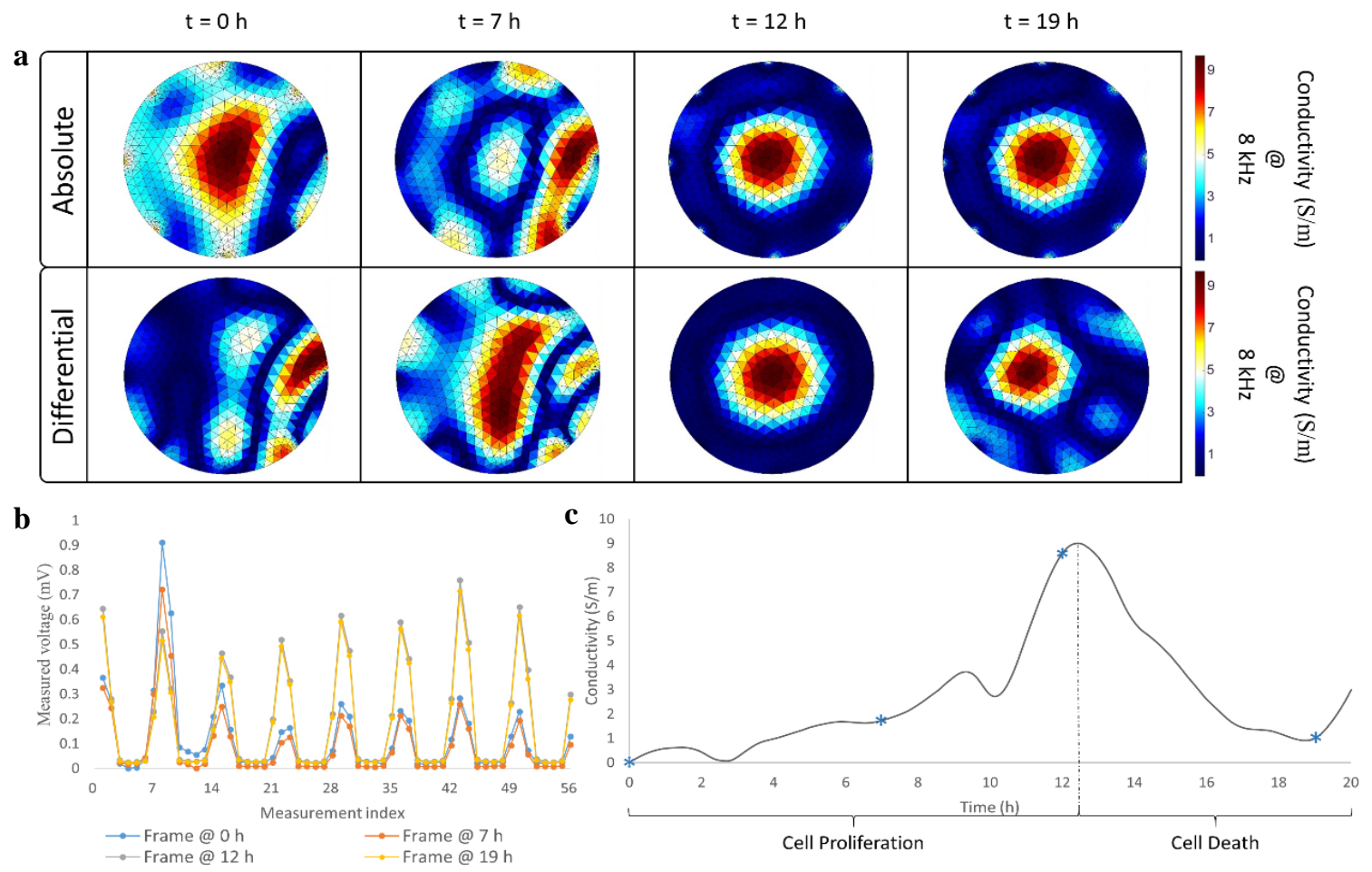

Figure 9 (a) The EIT image at $t=0 \mathrm{~h}$ shows the starting point of the growing curve in function of time. The progression of the growth curve is show at several intervals: $0 \mathrm{~h}, 7 \mathrm{~h}, 12 \mathrm{~h}$ and $19 \mathrm{~h}$. (b) Besides the images itself an overview of the measured voltages is shown for each frame. (c) Taking the mean value of each frame and plotting these values for the total time span of $20 \mathrm{~h}$ results into the following chart. A clear trend is shown during the proliferation phase followed by the phase of cell death.

The first way of monitoring the cellular growth of the yeast cells in function of time describes an iterative process of generating a conductivity matrix solely based on the measurements of boundary electrodes. By performing this technique only the subject under test needs to be measured, only the data collected from this measurement can be fed into the algorithm to generate the images. A disadvantage when using this technique is the small changes in the measured voltages, so trying to find the optimal solution for this will be very difficult.

A second approach is to take the differential measurement and feed this data into the EIT algorithm. By subtracting the measured data from a reference measurement subtle changes in the voltage measurements on the boundary electrodes will show more distinct changes and this results into a more accurate image. Other advantages include the exclusion of the contact impedance and errors in the electrode position.

The results show similarities between the images when using the absolute or the differential values as an input for the solver. When looking at the range of conductivity the medium shows a value of $3 \mathrm{~S} / \mathrm{m}$ and lower, this however in some images include noise. The conductivity values of the growing yeast is between 3 and $9 \mathrm{~S} / \mathrm{m}$, this verifies the earlier experiment where the impedance spectrum is taken from both agar and a combination of agar and yeast. These results showed that the yeast gives a lower resistance value than the medium, this by extend concludes the EIT results where the yeast has a higher conductivity than agar. When the mean value of every image is taken based on the differential method, the progression of the growth of yeast can be monitored in function of time. In figure (9) two phases are visible in this process. First there is the proliferation phase, in this phase a clear increase of conductivity can be established, the values start close to $0.1 \mathrm{~S} / \mathrm{m}$ and increase until $9 \mathrm{~S} / \mathrm{m}$. The second phase is the death of the yeast cells, a consequence is the decrease of conductivity from $9 \mathrm{~S} / \mathrm{m}$ to $1 \mathrm{~S} / \mathrm{m}$. Changes in the membrane potential values can be explained by the small volume of a cell, large surface-to-volume ratio and high transport capacity of proteins through the proton pump in the cell barrier. These things all contribute to the ion homeostasis in a yeast cell [21].

5 Conclusion The focus of this paper is to execute a feasibility study on how EIT imaging can help in the analysis of the cell-drug interaction. This is done by using existing hardware in combination with a custom sensor and EIT algorithm. This results into a working miniaturised experimental setup for electrical impedance tomography, a noninvasive and contactless imaging technique. A circular sensor array of 8 electrodes gives the possibility to detect cellular growth at any location within this region. In addition to the solver a script is written to cross-validate the different parameters that are needed to reconstruct the most accurate image. A spatial reconstruction of the outline is made regarding the growth of yeast by using the adjacent method. 
However, to this day mostly the differential method is used in (bio)medical applications, these results show that the correct model together with the correct parameters give comparable results. Nevertheless, differential measurements will result into much more stable measurements because of the elimination of electrode position errors, contact impedance and also a major advantage of differential measurements is the speed of reconstruction. Based on these results it is possible to verify the feasibility study regarding the development of a EIT sensor to monitor cellular growth in function of time.

Acknowledgements This work is funded by the wearIT4health project which is carried out under Interreg V-A Euregio Meuse-Rhine and is supported by the European Union and The European Regional Development Fund and with financial support of province of Limburg - Belgium.

\section{References}

A. Adler, M. B. Amato, J. H. Arnold, R. Bayford, M. Bodenstein, S. H. Böhm, B. H. Brown, I. Frerichs, O. Stenqvist, N. Weiler, G. K. Wolf, "Whither lung EIT: Where are we, where do we want to go and what do we need to get there?," Physiol. Meas., vol. 33, no. 5, pp. 679-694, 2012.

[2] A. V Naumova, M. Modo, A. Moore, C. E. Murry, and J. A. Frank, "Clinical imaging in regenerative medicine," Nat. Publ. Gr., vol. 32, no. 8, pp. 804-818, 2014.

[3] H. Ha, M. Konijnenburg, B. Lukita, R. Van Wegberg, J. Xu, R. Van Den Hoven, M. Lemmens, R. Thoelen, C. Van Hoof, N. Van Helleputte, “A Bio-Impedance Readout IC with Frequency Sweeping from $1 \mathrm{k}-$ to- $1 \mathrm{MHz}$ for Electrical Impedance Tomography,” Symp. VLSI Circuits Dig. Tech. Pap., 2017.

[4] A. R. Abdur Rahman, D. T. Price, and S. Bhansali, "Effect of electrode geometry on the impedance evaluation of tissue and cell culture,” Sensors Actuators, B Chem., 2007.

[5] P. Linderholm, L. Marescot, M. H. Loke, and P. Renaud, "Cell culture imaging using microimpedance tomography,” IEEE Trans. Biomed. Eng., vol. 55, no. 1, pp. 138-146, 2008.

[6] T. K. Bera and J. Nagaraju, “A MATLAB-Based Boundary Data Simulator for Studying the Resistivity Reconstruction Using Neighbouring Current Pattern,” J. Med. Eng., vol. 2013, pp. 1-15, 2013.

[7] T. K. Bera and J. Nagaraju, “A reconfigurable practical phantom for studying the 2D electrical impedance tomography (EIT) using a FEM based forward solver," Imaging, no. September, pp. 10-11, 2002.

[8] T. J. Yorkey, J. G. Webster, and W. J. Tompkins, "Comparing Reconstruction Algorithms for Electrical Impedance Tomography,” IEEE Trans. Biomed. Eng., vol. BME-34, no. 11, pp. 843-852, 1987.
[9] B. Brown and A. Seagar, "The Sheffield data collection system,” Clin. Phys. Physiol. Meas., vol. 8, no. 91, 1987.

[10] T. K. Bera and J. Nagaraju, "Studying the resistivity imaging of chicken tissue phantoms with different current patterns in Electrical Impedance Tomography (EIT)," Meas. J. Int. Meas. Confed., vol. 45, no. 4, pp. 663-682, 2012.

[11] T. Sun, S. Tsuda, K.-P. Zauner, and H. Morgan, “On-chip electrical impedance tomography for imaging biological cells,” Biosens. Bioelectron., vol. 25, no. 5, pp. 11091115, 2010.

[12] B. M. Graham, A. Adler, "Objective selection of hyperparameter for EIT.,” Physiol. Meas., vol. 27, no. 5, pp. S65-79, 2006.

[13] F. Braun, M. Proenc, J. Sol, J. Thiran, A. Adler, “A Versatile Noise Performance Metric for Electrical Impedance Tomography Algorithms,” vol. 9294, no. i, pp. 1-9, 2017.

[14] A. Adler, R. Guardo, "Electrical Impedance Tomography: Regularized Imaging and Contrast Detection,” vol. 15, no. 2, pp. 170-179, 1996.

[15] C. HANSEN, D. P. O'LEARY, "The Use of the L-Curve in the Regularization of Discrete Ill-Posed Problems," Soc. Ind. Appl. Math., vol. 14, no. 6, pp. 1487-1503, 1993.

[16] J. Nasehi Tehrani, A. McEwan, C. Jin, A. van Schaik, "L1 regularization method in electrical impedance tomography by using the L1-curve (Pareto frontier curve)," Appl. Math. Model., vol. 36, no. 3, pp. 1095-1105, 2012.

[17] G. H. Golubu, M. Heath, G. Wahba, "Generalized CrossValidation as a Method for Choosing a Good Ridge Parameter.”, vol. 21, no. 2, pp. 215-223, 1979.

[18] A. Adler, W. R. B. Lionheart, "Uses and abuses of EIDORS: an extensible software base for EIT,” Physiol. Meas., vol. 27, no. 5, pp. S25-S42, 2006.

[19] J. Liu, L. Ling, G. Li, “A novel combined regularization algorithm of total variation and Tikhonov regularization for open electrical impedance tomography.," Physiol. Meas., vol. 34, no. 7, pp. 823-38, 2013.

[20] M. Cheney, D. Isaacson, J.C. Newell, S. Simske, J. Goble, "NOSER: An Algorithm for Solving the Inverse Conductivity Problem,” vol. 2, no. 1990, pp. 66-75, 1991.

[21] V. Volkov, "Quantitative description of ion transport via plasma membrane of yeast and small cells," Front. Plant Sci., vol. 6, no. June, pp. 1-20, 2015. 